residues indexed according to their $\mathrm{C}, \mathrm{H}$ content, and in the "Permuted Formula Index" the rarer elements are emphasised and 7,842 entries are grouped under 70 rare-element subheadings. The "Author Index" has 43,039 citations to 10,362 authors ordered alphabetically by surname. The "Literature Index" has 15,933 citations from 260 journals, conference reports, and so on.

The "Compound Name Indexes" are valuable sources for the reader who wishes to discover what crystallographical results are available for particular types of organic or organometallic compounds. In the index of organometallic compounds and metal complexes, each subsection gives a comprehensive survey of crystallographic studies involving a particular metal and references to specific ligands can be obtained by working through the subsection. I quickly obtained 82 references to cobalt phosphine complexes, for example. In the organic index, I looked for bicyclo $[3,3,1]$ nonane, cyclononane and cyclodecane derivatives and analogues and obtained 22, 6 and 30 references, respectively. A typical entry in this section has the form:

1,5-diaza-6,10-Cyclodecadione . . . 7 34. 8

\section{Dream of classical thermodynamics}

The Concepts and Logic of Classical Thermodynamics as a Theory of Heat Engines. By C. Truesdell and S. Bharatha. Pp. 151. (Springer: New York, Heidelberg and Berlin, 1978.) DM56.30; \$28.30.

IN this book we pass from the elements of thermodynamics to the second law by methods and concepts which (the author assures us) were known in 1854, if only someone had had the insight to string the relevant ideas together. The mathematics is rigorous, and definitions, axioms, scholia, lemmas and corollaries abound. The text is furthermore spiced with critical and historical remarks regarding earlier treatments. Authors of textbooks in general are taken to task in no uncertain terms, for example, for not using the calculus (p. xv), or for appealing to "the absurdity" of perpetual motion machines "when reason fails" (p. xii). Now Truesdell is himself a considerable textbook writer if we recall Thermodynamics for Beginners (1966) and Rational Thermodynamics (1969), and it could be that he is here condemning his own earlier books, along with those

where 7 specifies the MSD volume and 34 the compound class. On turning to the "Literature Index" the appropriate entry is:

\section{34. 8 Acta Crystallogr. \\ Sect.B . . .31 137275}

Here, 31 is the journal volume, 1372 is the initial page of the article and 75 is the year of publication (1975). Natural products with particular molecular features cannot normally be located by the keyword approach, as such compounds usually have trivial, rather than systematic, names. Accordingly, the list of cyclodecane derivatives and analogues did not include any germacranolide sesquiterpenoids and these have to be found by looking for particular compounds by name (for example, elephantol, glaucolide $\mathbf{A}$, and so on) or by listing and examining all sesquiterpenoids (compound class 53).

The book is well produced and very reasonably priced for the wealth of information it provides in about 660 large pages ( $9 \times 12$ inches). It will be a welcome addition to libraries and laboratory desks. A minor flaw in the review copy is faulty printing of the final column in page L62 of the "Literature Index".

George Sim

George Sim is Gardiner Professor of Chemistry at the University of Glasgow, UK.

of others. One cannot be sure. The book is certainly more explicit about others: "In its hidden assumptions and vagueness, Sommerfeld's treatment is typical of those found in textbooks" (p49) or "Although ... Clausius somehow fell upon the right answer, his reasoning is mainly of the physical or metaphysical kind and does not carry conviction to a critical student" (p147).

The night after I read the book I dreamt that I just entered a lofty hall which looked almost like a church. I recognised many people in the small congregation as textbook writers on thermodynamics from the present and the last century. A fiery speakerclearly one of the authors-was just finishing an address "and thus our subject is a 'congenitally crippled science' (p.x), and (pointing his finger) you are to blame". There was agitation as he sat down and the Chairman, who I felt sure was Willard Gibbs, remarked in measured tones that a provocative speech, though not his way, was a stimulating experience. It fell to an elegant 1904 author, G. H. Bryan, to summarise the scientific content of the book. He pointed out that we are dealing with a system in two variables which, in a usual notation are $v$ and $t$. We assume $p=p(\nu, t)$ and $(\delta p / \delta v)_{\mathrm{t}}<0$ (axiom I, p8); $\mathrm{d} Q=C_{v} \mathrm{~d} t+l_{v} \mathrm{~d} v$ where $C_{\mathrm{v}}>0$ and $l_{\mathrm{v}}$ are appropriate functions (axiom II,, 30$)$. The existence of a function $W\left(t^{\prime}\right.$, hot $\left.t_{\text {cold }}, Q_{\text {hot }}(C)\right)>0$ for the work done in Carnot cycle $C$ is axiom III, p67, and that this function is the same for all bodies and invariant under a change of temperature unit gives the remaining axioms IV (p129) and V (p140). Normal thermodynamics can thus be constructed. "The historical remarks", he added with a smile, "mean that some extra space is needed." Thus, that $l_{v}=p=N k t / v$ for an ideal gas is called Holtzmann's assumption, and that $C_{p}-C_{v}=N k$ for such systems is called Mayer's assumption". In a brief discussion the chairman pointed out that the number of particles was clearly kept constant in the system, Nernst remarked that the absolute zero was not introduced, and Born pointed out that by having two variables with continuity assumptions the Pfaffian form for d' $Q$ has always an integrating factor so that some interesting thermodynamics was left out. In reply, the authors reiterated that their objective was to reach 1854 , but to reach it with precision and historical footnotes. (There was a grumble from someone that footnotes for other than references was a sign of sloppy writing.)

Gibbs then introduced a discussion on the likely pedagogical success of the book. It was Sommerfeld's opinion that the mixture of mathematical arguments and sophisticated historical points concerning the thermodynamics of Carnot (itself a controversial problem), Clausius and Reech made the book hard for beginners. "The flouting of advice of international commissions on the use of scientific symbols makes it equally hard for those who know the subject", remarked Guggenheim. "What is one to make of the use of $K_{p}$ and $K_{v}$ not for compressibilities but for heat capacities?", he asked. "Are we then agreed it is useful for supplementary reading by young and old alike?", urged Gibbs. There was embarrassed silence. At last Lord Kelvin rose. "It is a contribution to our science, to which we have devoted our lives; it should be read and studied. But I would not like to think", he added with a twinkle, "that any young whipper-snapper can come along, do a bit of axiomitising of science and then look down his nose at those who expounded and created it before him. Generosity in science and in acknowledging indebtedness in science is a more important lesson to teach than entropy or energy". The applause turned into the low notes of my alarm clock, and I have written down here what I had dreamt. P. T. Landsberg

P. T. Landsberg is Professor of Applied Mathematics at the University of Southampton, UK. 